\title{
Glassless Augmented Display for Public Signage
}

\author{
Akihiko Shirai \\ Kangawa Institute of \\ Technology 1030 \\ Shimoogimo,Atsugi City, \\ Kanagawa Prefecture,Japan
}

\author{
Hisataka Suzuki \\ Kangawa Institute of \\ Technology 1030 \\ Shimoogimo,Atsugi City, \\ Kanagawa Prefecture,Japan \\ Kazuhisa Yanaka \\ Kangawa Institute of \\ Technology 1030 \\ Shimoogimo,Atsugi City, \\ Kanagawa Prefecture,Japan
}

\author{
Yuta Yamaguchi \\ Kangawa Institute of \\ Technology 1030 \\ Shimoogimo,Atsugi City, \\ Kanagawa Prefecture,Japan
}

\begin{abstract}
This project realizes the spatial augmented reality in the public. Audiences can watch their preferred images and audios on the public signage. It is high applicative technology cause, it is configured by the consumer based $4 \mathrm{~K} \mathrm{LCD}$, super-directive speakers, lenticular lens and Multi-view Images Synthesizer implemented into an Unity application. It enlarges virtuality, without any glasses and/or developmental hardwares for user side, into the public space like stadiums, parks, public halls and restaurants and used for displaying various contents such as sports games and concerts by audience's movement.
\end{abstract}

\section{CCS Concepts}

-Computing methodologies $\rightarrow$ Mixed / augmented reality; -Hardware $\rightarrow$ Displays and imagers; •Information systems $\rightarrow$ Multimedia content creation;

\section{Keywords}

Multiplexed display; Auto-Stereo; lenticular; spatial augmented reality

\section{MOTIVATION SPATIAL AUGMENTED RE- ALITY IN THE PUBLIC}

In recent years, the usage of large screen and digital signage has expanded rapidly in the public. These public screens are usually placed in stadiums, public halls, airports, and transportation terminals displaying not only game results and arrival/departure information but also commercials and sponsor messages. It is essential for travelers to be

*gad@shirai.la, +81-46291-3191

Permission to make digital or hard copies of all or part of this work for personal or classroom use is granted without fee provided that copies are not made or distributed for profit or commercial advantage and that copies bear this notice and the full citation on the first page. Copyrights for components of this work owned by others than the author(s) must be honored. Abstracting with credit is permitted. To copy otherwise, or republish, to post on servers or to redistribute to lists, requires prior specific permission and/or a fee. Request permissions from permissions@acm.org.

VRIC 2016 Laval, France

(C) 2016 Copyright held by the owner/author(s). Publication rights licensed to ACM. ISBN 978-1-4503-4180-6/16/03 .. \$15.00

DOI: http://dx.doi.org/10.1145/2927929.2927930 able to see their departure and arrival schedule as well as the commercials. Because of this it would be beneficial to display information either in a static format or in an animated format depending on the situation and types of audiences viewing the public screen as different people have different tastes. To solve this complex display issue we propose a new system which can solve this problem by combining our own configured glassless 3D display technology with a superdirective speaker technology.

Up to now 3D display devices had been wider spread, but haven't really existed killer application especially for the public screen. This project is named as "Glassless Augmented Display (GAD)" which proposes a new display system in which multiple virtual screens are produced on a single physical screen. This technology is based on a current glassless 3D stereo graphics technology. It can present different information for many and unspecified people at the same time by combining a $4 \mathrm{~K}$ display device with a lenticular lens plate and a Multi-view Image Synthesizer (MIS) running on Graphics Processing Unit (GPU). It can present multiple channels simultaneously without any wearable device such as stereo glasses for the viewer side. Efficient information presence application like augmented reality, multi-language and/or multi-directional will be possible in theater or living room but also in the public space for any visible user. It is possible to watch a completely different image by viewing position interactivity.

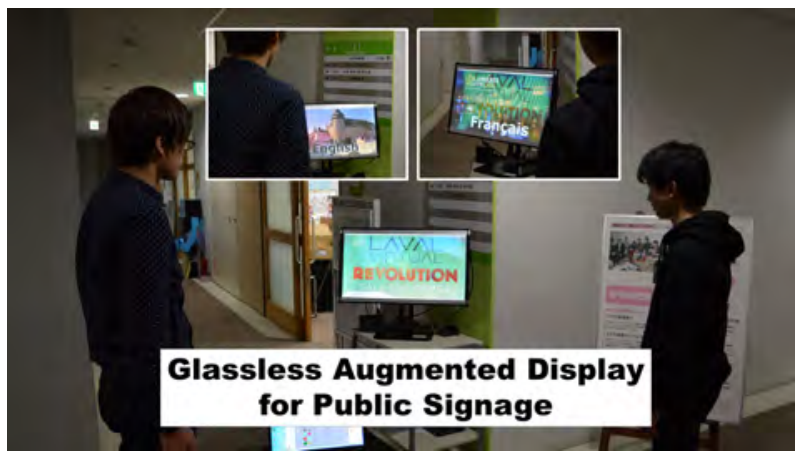

Figure 1: Prototype of proposing glassless augmented display for public signage. Each viewer can see different images and audios from different directions. 


\section{RELATED WORKS}

Bimber and Rasker proposed "Spatial Augmented Reality" in 2005[3], the technologies extend projector utilization like projection mapping. "Invoked Computing"[12], which is configured projector, camera, parametric speaker and steering base ,in Laval Virtual ReVolution 2011 is an ideal realization of ubiquitous computing. However hard registration is needed for the environment and moving projectors and the target user must be alone or limited. Shirai proposed multiplex-hidden technologies which can realize multiple channels on a screen and it continues to 4th generation using projectors and flat panels[8]. However, the multiplexhidden technology is based on polarization and color cancelling. Audiences need a filter to choose their preferred contents. The idea using autostereo display to realize spatial augmented reality seems not a really new idea. In 1692, Gaspar drew "Bois Clair", which can see different images by 2 sides[2]. In the late 1930's, Vari-Vue succeed multiple image lenticular image was formed and this was the seed that started the Vari-Vue company. During this time, Vari-Vue coined the name "lenticular" [1]. Fujitsu Ten, "AVN7905HD"[9] and Mercedes Benz "SPLITVIEW"[5] are applied similar technology to car navigation system for 2 sides like driver and assistant seats. Sharp Inc. proposed "triple view LCD"[7] with parallax barrier to realize 3 channels with viewers' orientation. In this system, different contents can be viewed from different viewpoints such as from a driver seat perspective or from a backseat's perspective. However, these techniques are not scalable because it is specialized in the application to a car. They are not always suitable for ourcurrent application field of digital signage. It requires high resolution, interactive and/or moving picture, larger screen with less cost even in brighter space for various audiences. Our challenge is extending public displays virtually by our scalable LFD method to realize Glassless Augmented Display for multi directional viewers.

\section{METHOD}

The proposed system consists of a video subsystem and an audio subsystem as shown in Figure 2. The video subsystem is a part where a number of virtual video channels are achieved by using a single real display. The audio subsystem is the part where many different sound waves are radiated from super-directivity parametric speakers to different directions.

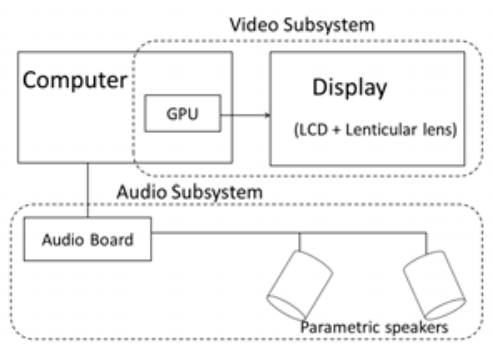

Figure 2: System configuration.

\subsection{Video Subsystem}

Our proposed method is common with configuring a light field display (LFD) consisting of a liquid crystal display (LCD) and a lenticular lens panel as shown in Figure 3.
A transparent acrylic plate of the foreground is used to fix the lenticular lens panel tightly.

LFD utilizing a LCD is often used for autostereoscopic three-dimensional (3D) display because the 3D image is bright and clear. In case of a 3D display, two or more images which were captured from slightly horizontally different camera positions are synthesized, and the resulting image, usually called a lenticular image, is obtained. When the lenticular image is displayed on the LCD and observed through a lenticular lens which is put just above the LCD, a stereoscopic image is seen. In the case of $3 \mathrm{D}$ display, each view corresponds to one of the images used for synthesizing the lenticular image. Although the quality of the 3D image decreases when a cross talk occurs among different views, it is not a serious flaw because views are usually mutually similar, especially in the case of multi-view autostereoscopic displays.

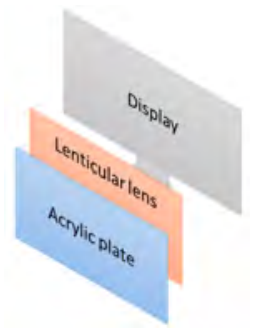

Figure 3: Display configuration.

On the other hand, it is highly probable that completely different contents, for example a sports program, a music program and a news program, are displayed in each view in the proposed system. If there is a cross talk, unrelated contents are mixed and users would feel unconfortable. Since it should be avoided, more precise optical system is required.

Figure 4 shows a sectional view of a simple conventional LFD using a lenticular lens, which is an array of semicylindrical lenses. Red, green and blue subpixels are paced just below the flat side of the lens sheet. The light that came out of each subpixel becomes almost parallel beam because the focal length is usually almost equal to the thichness of the lens sheet. In Figure 4 there are eight subpixels per a lens pitch. Therefore, the number of the direction in which light travels is eight. Thus the number of views is also eight. Because eight is an integer, this system is called the integer view method.

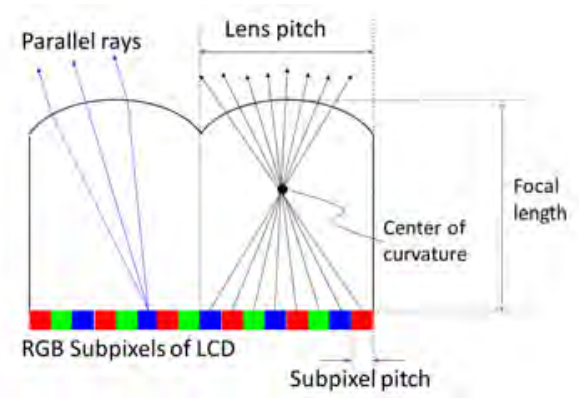

Figure 4: Integer view method.

However, when off-the-shelf LCD and ready-made lenticular lens are combined to produce a LFD, the possibility that 
the ratio of the pixel pitch and the lens pitch is exactly an integer number by chance is almost zero. In most cases it becomes non-integer, or "fractional" value. Even in this case a LFD can be produced by using Ishii's "fractional view" method [4,6]. Attached lenticular panel gives a non integer (fractional) light direction sets from subpixels. Afterward, Ishii's method was extended by Yanaka to cope with the integral photography which has both horizontal and vertival parallax $[10,11]$. The display system we adopted in this project falls into the category of the fractional view since it is assumed that non-integer number of pixels exist per lens pitch. However, the amount of calculation is being reduced substantially by replacing the time-consuming ray-tracing process from each subpixel to choosing the most appropriate rendered image from a number of pre-rendered images. In the fractional view system, very unsightly moire may occur since the lens pitch and the subpixel pitch is not an integer ratio. Therefore we slanted the lenticular lens panel against the LCD as shown in Figure 5. Moire became less visible because it was transferred to high frequency band, and the subjective image quality was improved substantially. Conventionally, slanted lenticular system has been adopted in multi-view 3D display systems. However the purpose of slanting is to increase the number of view in this case, and the angle of slanting have to be a specific angle obtained theoretically. On the other hand, the angle of inclination for the reduction of the moire has considerable width of acceptance. We tilted the lenticular lens just 10 degrees in the experiment described later. Where

$$
\text { HorizontalLensWidth }=\frac{\text { OriginalLensWidth }}{\cos (\theta)}
$$

The total number of view is assumed to be three in Figure 5 so that the figure becomes easy to understand, although the actual number of view is 12 in the experiment described later. Since the number of views is three, each of the semicylindrical lens is divided into three areas. Which view each subpixel indicates is decided by which area the center of the sub-pixel belongs to. Number from 1 to 3 on each subpixel shows a view to which the subpixel belongs. Very high processing capability is required in order to synthesize plural video signals in real time. Therefore we decided to carry out it with a graphics processing unit (GPU).

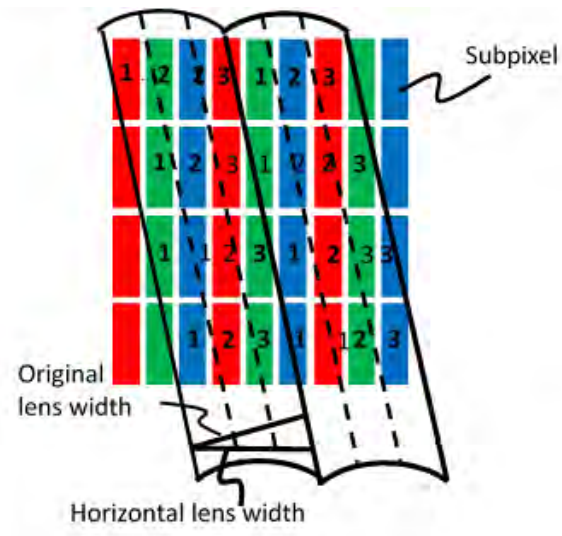

Figure 5: Slanted lentivular system for moire reduction.

\subsection{Audio Subsystem}

In this system, not only to present different images and videos in different directions, the acoustic associated with them must also be presented. Therefore we use parametric speakers which have very high directivity. Since video and audio signals must be synchronized, they are output from the same PC by using Unity, which is a very popular game engine.

\section{EXPERIMENT}

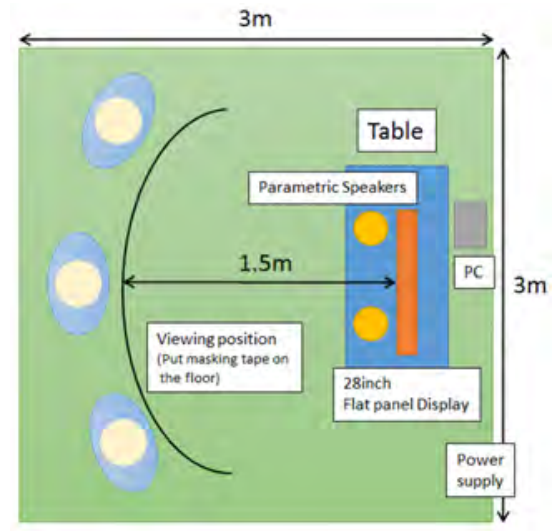

Figure 6: Installation of the system.

Figure 6 shows the installation of the system. In the experiment, audiences can see different visual contents given to 3 directions and two different audios accompanying them.

\subsection{LCD and Lenticular Lens}

In the experiment, IIYAMA ProLite B2888UHSU, which is a 28 inch $4 \mathrm{~K}$ (3840x2160pixels) LCD, was used among various $4 \mathrm{~K}$ displays which are widely distributed in the market. A 15 line per inch (LPI) plastic lenticular lens was used among products easily available in the market. The main specifications of the LCD and its relation to the lenticular lens are shown in Figure 7. The value of field per lens shown in this figure is the theoretical value of the case without the subpixel rendering. When a subpixel rendering is performed, the value becomes triple.

\subsection{Parametric Speakers}

Different sounds are played by multiple parametric speakers (Switch Science, SSCI-018425) turned to different directions. As a result it was confirmed that there was a sufficiently high directivity since the mixture of the sounds did not occur.

\subsection{Overall Operation of the System}

We have created a content for the test of the overall operation as shown in Figure 8. It is confirmed that a still image is visible when viewed from the canter, and that a French video and an English video are visible when viewed from the left and right respectively. Sounds which are attached to the two videos were able to be heard without mixing.

Therefore it became apparent in the experiment that audiences can see different contents and audios which is given to 3 directions. The system can provide interaction, however, in our testing, audience stop and see their preference 


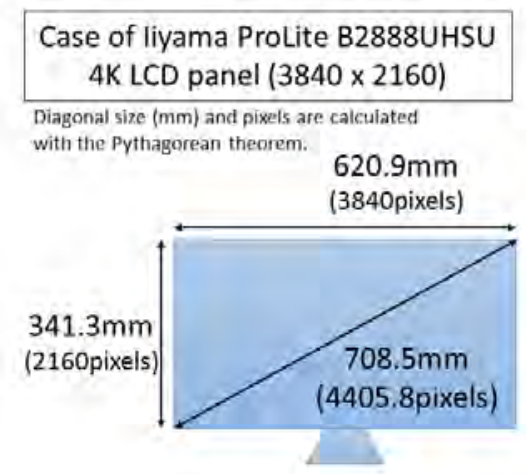

When using 15lpi lenticular, Each lens size is $1.69 \mathrm{~mm}$. Fields per lens $=\frac{1.69 \mathrm{~mm}}{0.1608}=10.5099$ fields $/$ lens

Figure 7: Main specifications of the LCD and its relation to the lenticular lens.

contents by their interest freely. Interactivity will be added until the actual demonstration in Laval Virtual ReVolution 2016. As an expectable contents, we can propose multi language signage in Laval Virtual for non-French speaker visitors. As another demonstration, we can propose "visitor survey" with 3 sides to share and visualize audience impressions on the site. Other idea from organization are welcome.

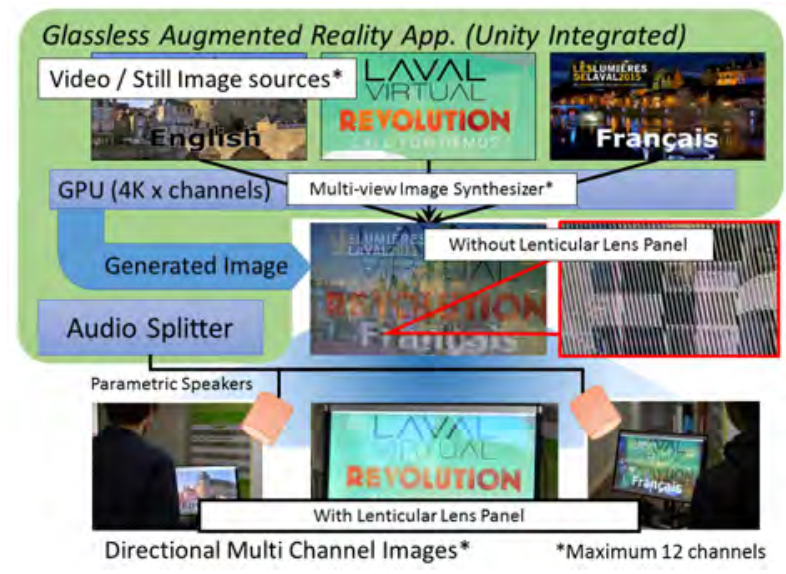

Figure 8: Glassless Augmented Display and system configuration.

\section{CONCLUSION}

A new multimedia display system consisting of a multidirectional display and super-directive speakers has been proposed and its prototype has been produced by using a high-resolution (4K) LCD and a lenticular lens. Different visual contents and accompanying audios are played simultaneously, and each user can choose his/her preferred contents by simply changing the viewing position by walking left and right in front of the display. The system is highly applicable because all software system are integrated into a Unity application.
In a conventional digital signage, there was no freedom of choice to the audience because everyone have to see the same content. On the other hand, when viewed from the side that provides the information to general public, there was no means to provide the appropriate information according to the attributes of the viewer. It is highly probable that this situation can be drastically changed by the proposed system. A right person can receive now right information everywhere, for example in a park, in a stadium, in a public hall, in a station and in an airport, without the need for wearing special glasses. We would like to promote the development strongly, from both sides of technology and application.

\section{Acknowledgments}

This work was supported by JSPS KAKENHI Grant Numbers JP25330440, JP25330244.

\section{REFERENCES}

[1] Vari-vue: Inventor of the lenticular imaging technique. http://www.didik.com/vv_his.htm.

[2] Gaspar Antoine. Bois-clair, 1692. http://www. robertsimon.com/pdfs/boisclair_portraits.pdf.

[3] Oliver Bimber and Ramesh Raskar. Spatial Augmented Reality: Merging Real and Virtual Worlds. A. K. Peters, Ltd., Natick, MA, USA, 2005.

[4] Motonaga ISHII. Spatial image by fractional view display. Institute of Electronics, Information and Communication Engineers. EID, URCF2006, 106(338):33-38, nov 2006.

[5] Mercedes-Benz. Mercedes-benz introduces new splitview comand display for the s-class. http://www.emercedesbenz.com/Dec08/10_001531_ Mercedes Benz Introduces New SplitView COMAND Display For The S Class.html.

[6] Namco. Namco developed fractional view 3d display method (in japanese), 2004. https://bandainamcoent. co.jp/corporate/press/namco/50/50-043.pdf.

[7] SHARP. Sharp dual directional viewing lcds. http://www.sharp-world.com/products/device/about/ lcd/dual/index.html.

[8] Hisataka Suzuki, Rex Hsieh, and Akihiko Shirai. Expixel: Pixelshader for multiplex-image hiding in consumer 3d flat panels. In ACM SIGGRAPH 2014 Posters, SIGGRAPH '14, pages 63:1-63:1, New York, NY, USA, 2014. ACM.

[9] FUJITSU TEN. Press release: "two world first" - the eclipse car av \& navigation systems concept for the 39th tokyo motor show 2005, 2005. http://www. fujitsu-ten.com/release/2005/10/20051013_e.html.

[10] Kazuhisa Yanaka. Integral photography suitable for small-lot production using mutually perpendicular lenticular sheets and fractional view. volume 6490 , pages 649016-649016-9, 2007.

[11] Kazuhisa Yanaka. Integral photography using hexagonal fly's eye lens and fractional view. volume 6803, pages $68031 \mathrm{~K}-68031 \mathrm{~K}-8,2008$.

[12] A. ZERROUG, A. CASSINELLI, and M. ISHIKAWA. Invoked computing: Spatial audio and video ar invoked through miming. In Proceedings of Virtual Reality International Conference (VRIC 2011). 